\title{
AUMENTO DA EFICIÊNCIA ENERGÉTICA NA MOAGEM SECUNDÁRIA DO CONCENTRADOR I*
}

\author{
João Paulo Melo Silva ${ }^{1}$ \\ Paulo Sergio de Oliveira ${ }^{2}$ \\ Alexandre Camilo Leles de Figueiredo ${ }^{3}$ \\ Marcos Geraldo Guimarães ${ }^{4}$ \\ Flávio Thimótio da Silva \\ Marcos Gomes Vieira ${ }^{6}$
}

\section{Resumo}

No beneficiamento de itabiritos pobres $(\% \mathrm{Fe} \approx 44,0)$ um dos principais desafios para as usinas de tratamento é a produção de concentrado dentro das especificações de qualidade e com menor custo. Nas usinas de beneficiamento a moagem é a operação unitária com o maior custo. Além disso, a moagem secundária é a operação unitária na qual a granulometria do concentrado é adequada às especificações para a etapa de flotação cleaner e para o transporte em mineroduto. Dessa forma, o aumento da eficiência energética no circuito de remoagem permite reduzir os custos e aumentar a eficiência do circuito de flotação cleaner. Amostragens de rotina e testes de bancada realizados com amostras coletadas no concentrador I indicaram oportunidade de aumentar a eficiência energética $(\mathrm{kWh} / \mathrm{t})$ da remoagem e reduzir a granulometria na alimentação da flotação. Com esse objetivo, foram realizadas alterações no circuito de remoagem do pré-concentrado da flotação mecânica do concentrador I da Samarco. Essas alterações consistiram em moer todo o pré-concentrado em circuito fechado reverso e não mais em dois estágios, sendo um em circuito aberto e outro em circuito fechado. Essas alterações realizadas no circuito permitiram aumentar a eficiência energética do circuito de remoagem, reduzir o consumo energético em 9,3\% e adequar as granulometria e grau de liberação para a etapa subsequente de flotação.

Palavras-chave: Moagem; Tamanho de partícula; Flotação; Teor de $\mathrm{SiO}_{2}$ no concentrado.

\section{SECUNDARY GRIDING ENERGETIC EFICIENCE INCRISING AT CONCENTRATOR I Abstract}

In processing of itabirite with iron content of 44,0, a major challenge for the treatment plants is to product concentrate within quality specifications and at lower cost. In processing plants, grinding steps are the unit operation with the highest cost. Furthermore, the secondary grinding step is the operation which the size specification is suitable for the cleaner flotation stage and the pipeline transportation. In this way, increasing energy efficiency in the regrind circuit would reduce costs and increase the efficiency of cleaner flotation circuit. Routine sampling and bench tests carried out with samples collected at concentrator I indicated opportunity to increase energy efficiency $(\mathrm{kWh} / \mathrm{t})$ of regrind and reduce the particle size in the feed of flotation. For this purpose, the secondary grinding circuit at Samarco concentrator I was modified. These changes consisted in grinding all pre-concentrate in a reverse closed circuit. These changes have increased the energy efficiency of the regrind with a reduction of $9.3 \%$ in the energy consumption and adjust the particle size for cleaner flotation step.

Keywords: Grinding; Particle size; Flotation, $\mathrm{SiO}_{2}$ content on concentrate.

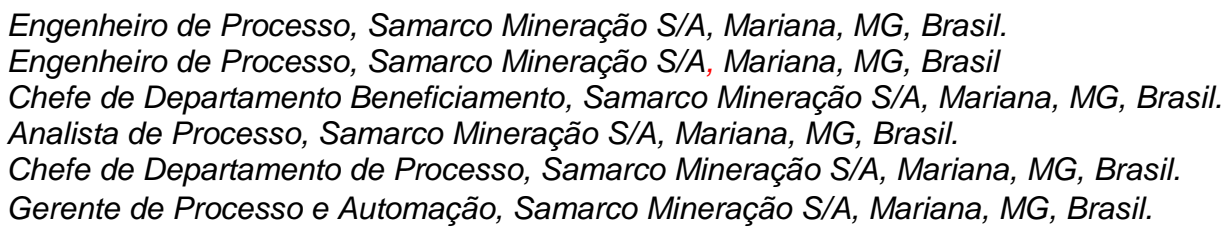




\section{INTRODUÇÃO}

Nas usinas de beneficiamento a moagem é a operação unitária com o maior custo. Sayadi et al [8] desenvolveram modelos de regressão com o objetivo de determinar os custos de capital (CAPEX) e custos operacionais (OPEX) para vários modelos de moinhos, como moinhos de bola e moinhos SAG. De acordo com os autores, as instalações de moagem representam $60 \%$ dos custos de capital e de $40 \%$ a $50 \%$ dos custos operacionais. Além disso, as instalações de moagem representam $60 \%$ de toda a energia consumida em usinas de concentração.

Segundo Jankovic e Valery [5] a maior eficiência de circuitos de moagem fechados se deve a dois fatores. O primeiro é a eficiência da etapa de classificação associado à etapa de moagem e o segundo é a existência da carga circulante. No entanto, é difícil mensurar o efeito de cada um destes fatores uma vez que os mesmos são relacionados. O aumento da carga circulante aumenta a capacidade do circuito de moagem e reduz a geração de finos. A imagem da esquerda da figura 1 apresenta a relação entre a carga circulante e a capacidade do circuito. Bond [3] definiu como $250 \%$ a carga circulante ótima considerando as limitações de eficiência na classificação em hidrociclones. Ainda segundo Jankovic e Valery [5], para um mesmo nível de carga circulante quanto maior a eficiência de classificação maior é a capacidade do circuito de moagem. Além disso, o aumento da carga circulante reduz a eficiência da etapa de classificação. A imagem da direita da figura 1 apresenta a relação entre a carga circulante, eficiência de classificação e a capacidade do circuito. Dessa forma os autores concluíram que o aumento na carga circulante pode aumentar a capacidade do circuito de moagem, no entanto a magnitude do aumento é limitado pela redução da eficiência de classificação, que é reduzida com o aumento da carga circulante.
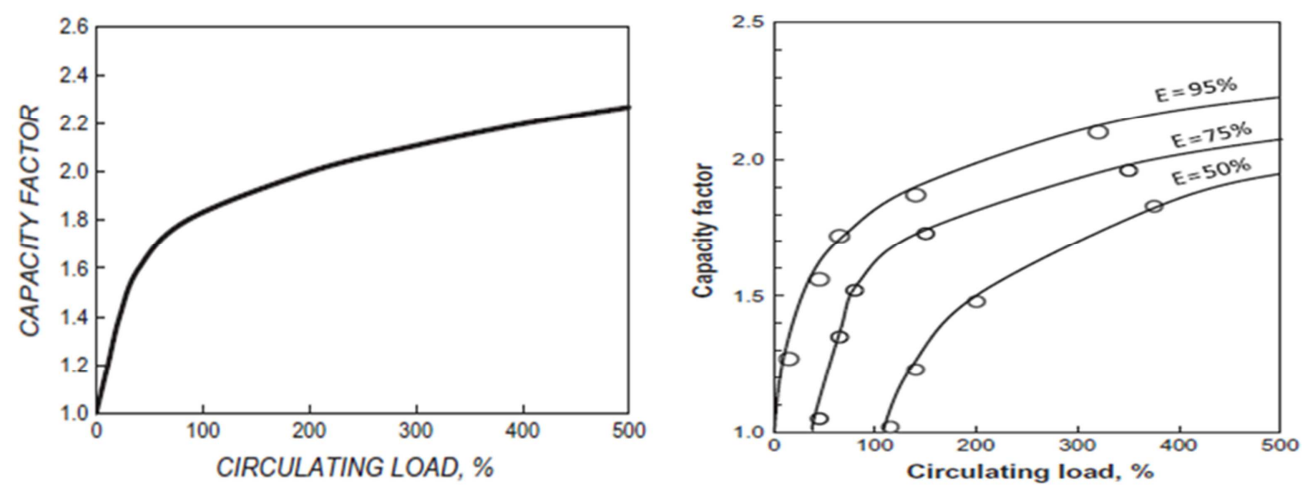

Figura 1. Relação da carga circulante, eficiência de classificação e a capacidade do circuito.

Powell e Ballantyne [2] também destacaram a influência da configuração do circuito de moagem (classificação + moagem) como um dos fatores que determinam a eficiência energética do circuito de moagem, ou seja, a relação de energia consumida por unidade de massa processada na moagem (kWh/t).

A moagem secundária é a operação unitária na qual a granulometria do concentrado é adequada às especificações para a etapa de flotação cleaner e para o transporte em mineroduto.

Pourghahramani e Dehgahani [7] propuseram a aplicação de um método para avaliação do desempenho de moinhos de bolas aplicados na etapa de remoagem considerando tanto a moabilidade quanto a flotabilidade do pré-concentrado. Segundo os autores, o principal objetivo da etapa de remoagem é promover a 
liberação das partículas mistas. Além disso, a lei de Bond não apresenta bons resultados quando aplicada a remoagem devido a dois fatores. $O$ primeiro se refere ao tamanho médio das partículas na alimentação do moinho e ao diâmetro dos corpos moedores aplicados na remoagem. O segundo fator se refere ao fato de que a lei de Bond não leva em consideração a razão entre a distribuição de tamanhos das partículas na alimentação dos moinhos e o diâmetro dos corpos moedores. A moabilidade do material foi avaliada considerando tanto a geração de finos quanto a liberação de partículas mistas. A flotabilidade foi avaliada considerando os resultados de qualidade do concentrado e recuperação metálica da alimentação e do produto da remoagem.

Chen et al [4] avaliaram o efeito do mecanismo de quebra durante a remoagem na etapa subsequente de flotação. A recuperação metálica na etapa de flotação se deve a quatro fatores: (I) tamanho das partículas; (ii) a geração de superfície específica; (iii) a oxidação das superfícies; (iv) a redistribuição do coletor na superfície após a remoagem. Os resultados obtidos mostraram que 0 fator predominante na determinação da recuperação metálica é a redistribuição do coletor na superfície das partículas. Após a remoagem, o coletor que foi adicionado na etapa de flotação antes da remoagem pode tanto se manter na superfície que já existia antes da remoagem quanto se adsorver nas superfícies geradas na remoagem. A figura 2 apresenta a distribuição do coletor considerando três mecanismos de quebra distintos (impacto, compressão, atrito). Como pode ser observado na figura, quando o mecanismo de atrito predomina ocorre maior geração de finos e o coletor se adsorve predominantemente nas partículas finas.

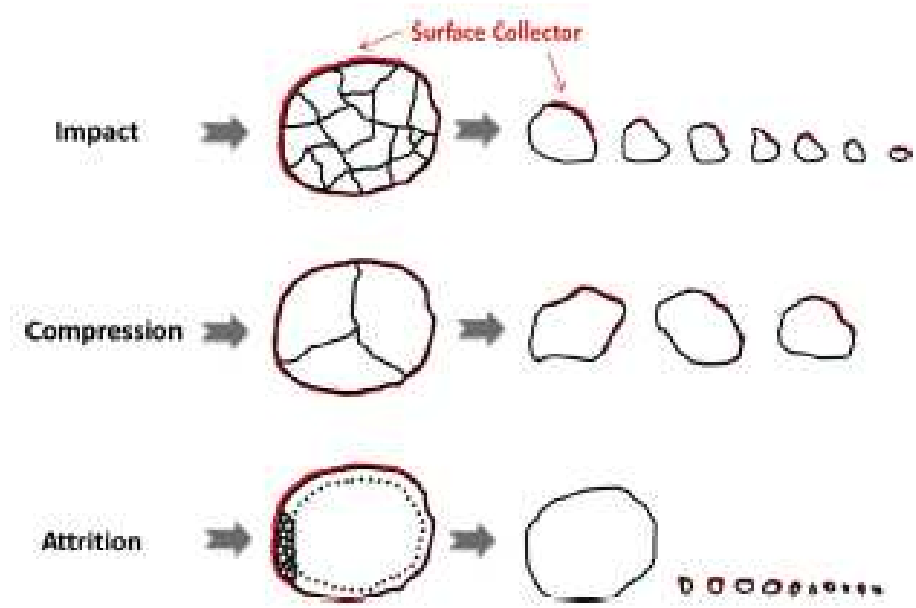

Figura 2. Distribuição do coletor considerando três mecanismos de quebra distintos (impacto, compressão, atrito).

Nadolski et al [6] desenvolveram um método para avaliar o desempenho em termos de eficiência energética da etapa de remoagem. O método consiste em comparar o consumo específico de energia (kWh/t) obtido no laboratório com o consumo específico de energia obtido na planta.

\section{MATERIAIS E MÉTODOS}

Para determinar a eficiência da etapa de remoagem do concentrador I da Samarco foram realizados testes de remoagem com amostras representativas da alimentação dos moinhos secundários. Os testes de remoagem foram realizados em moinhos de $0,2540 \mathrm{~m} \times 0,2540 \mathrm{~m}$, com velocidade de rotação igual a $65 \%$ da velocidade crítica e 
percentual de enchimento de $30 \%$. A massa da carga de bolas é de $18 \mathrm{~kg} \mathrm{com}$ o diâmetro de bolas variando de $15,0 \mathrm{~mm}$ a $30,0 \mathrm{~mm}$. A massa de sólido empregada no teste é de $3.500 \mathrm{~g}$ e o percentual de sólidos $(\mathrm{p} / \mathrm{p})$ é de $75,0 \%$. Os ensaios de remoagem consistem em realizar três testes nos seguintes tempos: 22 minutos, 44 minutos e 66 minutos. Para cada tempo de moagem foi medida a energia no eixo pinhão.

A energia requerida para etapa de moagem da amostra foi analisada e determinada segundo o modelo proposto por Donda [1]. Segundo o autor a energia específica necessária para reduzir a massa retida na malha de interesse é definida de acordo com a equação 2.1 .

$$
E=\frac{1}{K} \times \ln \left(\frac{F}{P}\right)
$$

$\mathrm{Na}$ equação 2.1, E é a energia específica em $\mathrm{kWh} / \mathrm{t}, \mathrm{K}$ (t/kWh) é o parâmetro característico do minério para a malha de interesse, $F$ é a massa de partículas na alimentação retida na malha de interesse e $P$ é a massa de partículas no produto retida na malha de interesse. O parâmetro $\mathrm{K}$ é determinado nos testes de moagem descritos acima.

Para avaliar o impacto da etapa de remoagem na etapa subsequente de flotação foram realizados testes de flotação em bancada com amostras da alimentação e produto da remoagem. Os testes de flotação em bancada foram realizados em uma célula de flotação WEMCO de $2.400 \mathrm{~mL}$, com rotação de $1.300 \mathrm{rpm}$. O depressor dos minerais de ferro utilizado foi o amido de milho (fubá) gelatinizado com hidróxido de sódio, na proporção mássica 5:1. O coletor empregado nos testes foi uma eteramina. As soluções de depressor, hidróxido de sódio e coletor foram preparadas nas seguintes concentrações: $1 \% \mathrm{p} / \mathrm{v}, 3 \% \mathrm{p} / \mathrm{v}$ e $1 \% \mathrm{p} / \mathrm{v}$, respectivamente. A massa de sólido empregada nos ensaios de flotação é de $1.500 \mathrm{~g}$ e o percentual de sólido $(\mathrm{p} / \mathrm{p})$ é 42,0\%. O depressor é condicionado durante cinco minutos e o coletor é condicionado durante um minuto. Para cada teste executado foi gerada uma amostra de concentrado e seis amostras de rejeito. As seis amostras de rejeito geradas se referem a seis tempos de flotação diferentes.

A análise do teor de ferro foi realizada por dicromatometria (método $\mathrm{TiCl} 3$ ). As análises químicas de sílica, alumina, fósforo e manganês foram realizadas em espectrômetro de emissão ótica com plasma acoplado indutivamente (ICPOES), da marca VARIAN, modelo 725-ES de propriedade da Samarco Mineração S.A. Os valores de perda por calcinação foram determinados por gravimetria (calcinação em forno mufla a $1000^{\circ} \mathrm{C}$ ).

\section{RESULTADOS E DISCUSSÃO}

Como pode ser observado na figura 03 (imagem da esquerda) o circuito de remoagem do concentrador I da Samarco era realizado em dois estágios. O primeiro em três moinhos $(5,2 \mathrm{~m} \times 10,4 \mathrm{~m} 4.325 \mathrm{kw})$ em circuito aberto. O segundo estágio também em três moinhos (5,2m x 10,4m 4.325kw) em circuito fechado. Modificando o circuito da forma que está representado na figura 03 (imagem da direita), os seis moinhos operam em circuito fechado. Com a modificação foi possível aumentar a eficiência energética do circuito de remoagem como pode ser observado na figura 04. A medida da eficiência energética do circuito de remoagem foi realizada comparando o consumo específico medido no laboratório de acordo o teste descrito 
no item 3 com o consumo específico obtido na planta industrial. Como pode ser observado no gráfico da figura 04, foi possível aumentar a eficiência energética do circuito de remoagem com redução de $9,3 \%$ no consumo de energia.
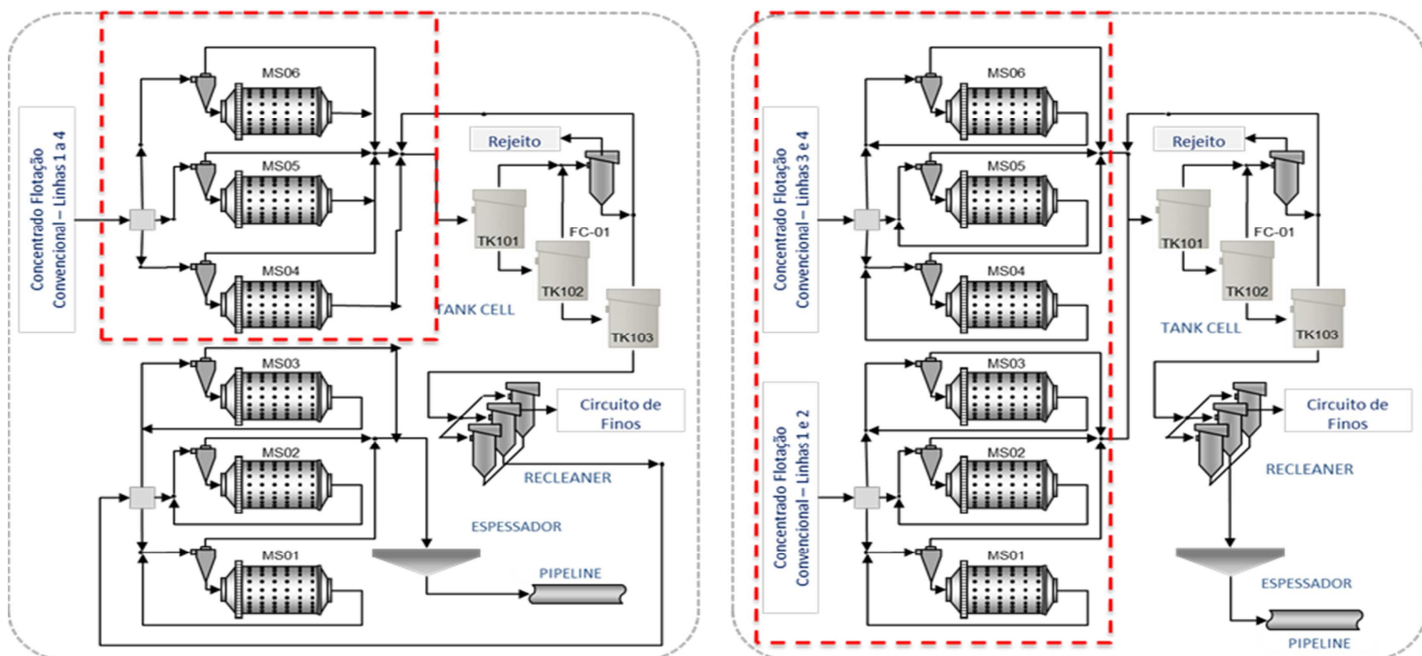

Figura 3. Fluxograma do circuito de remoagem e flotação cleaner antes e depois das modificações.

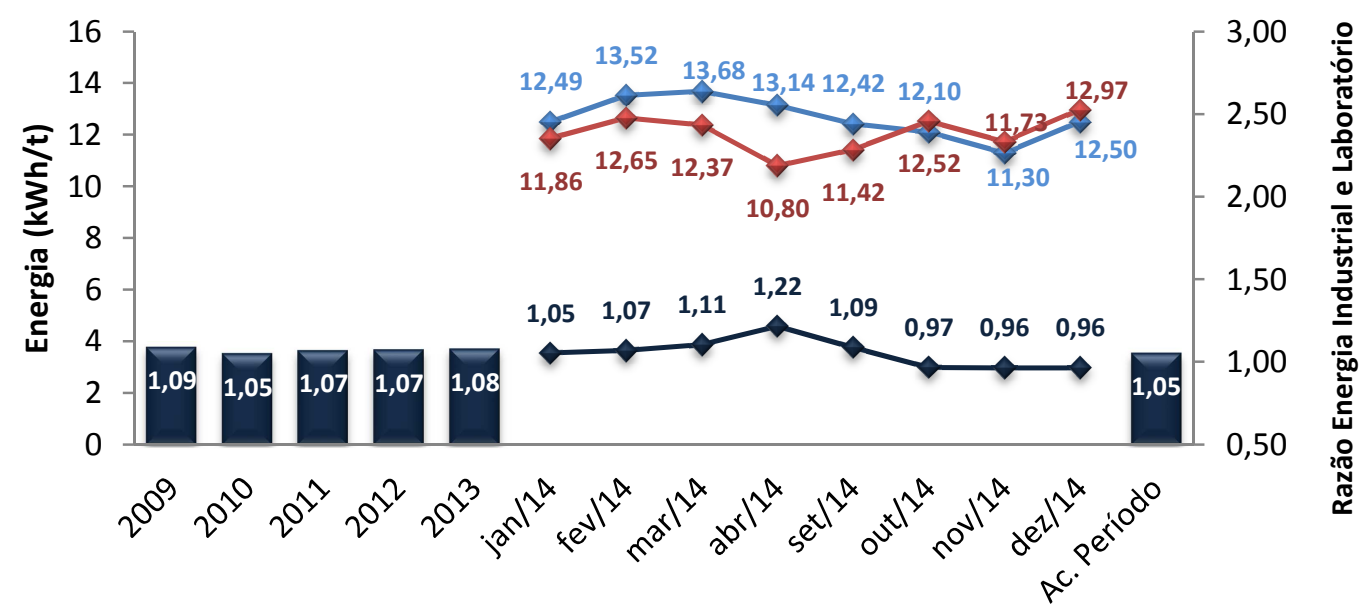

Razão E Ind./ Lab (Ano) حIndustrial $\prec$ Laboratório $\prec$ Razão E Ind. / E Lab

Figura 4. Consumo específico de energia medido no laboratório e medido na planta industrial.

As figuras 05 e 06 apresentam a distribuição granulométrica no underflow e overflow, respectivamente, dos ciclones secundários antes e após a modificação do circuito. Como pode ser observado nos gráficos, a modificação do circuito reduziu o tamanho das partículas no overflow dos ciclones secundários (alimentação da flotação cleaner) e aumentou o tamanho das partículas no overflow dos ciclones secundários (reduziu o percentual de material fino no interior do moinho). 


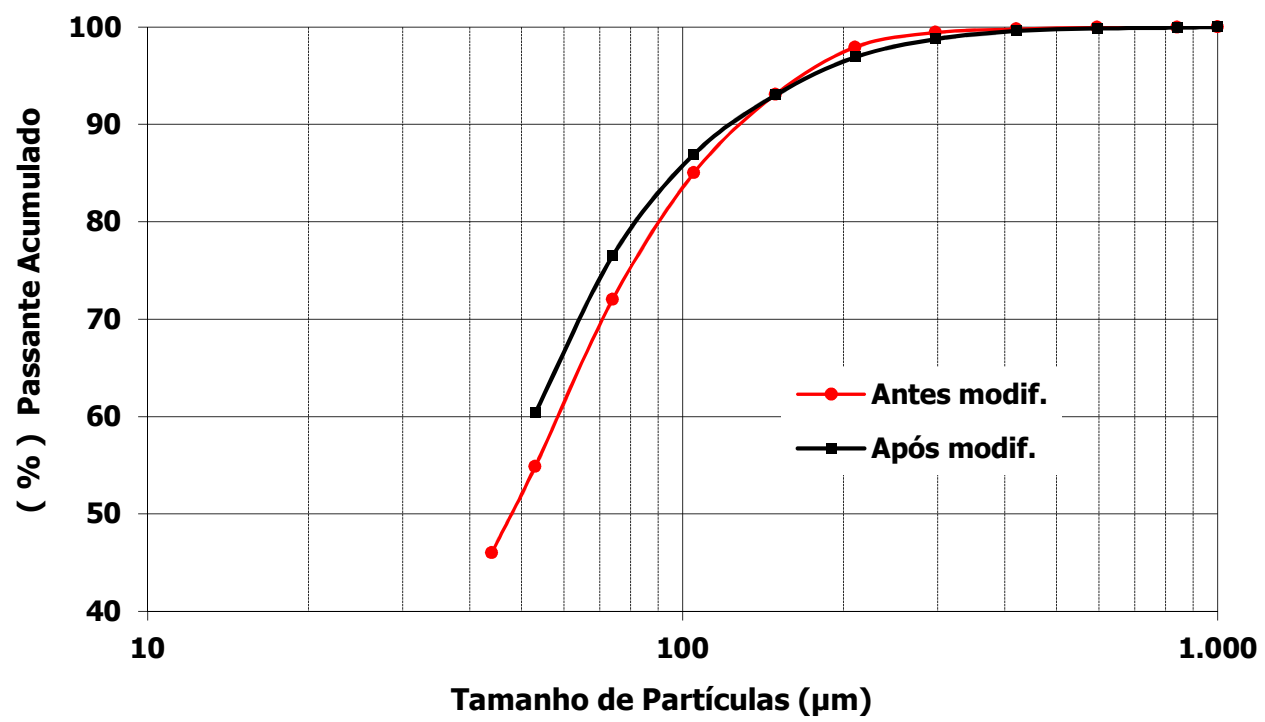

Figura 5. Distribuição granulométrica no underflow dos ciclones secundários antes e após a modificação do circuito.

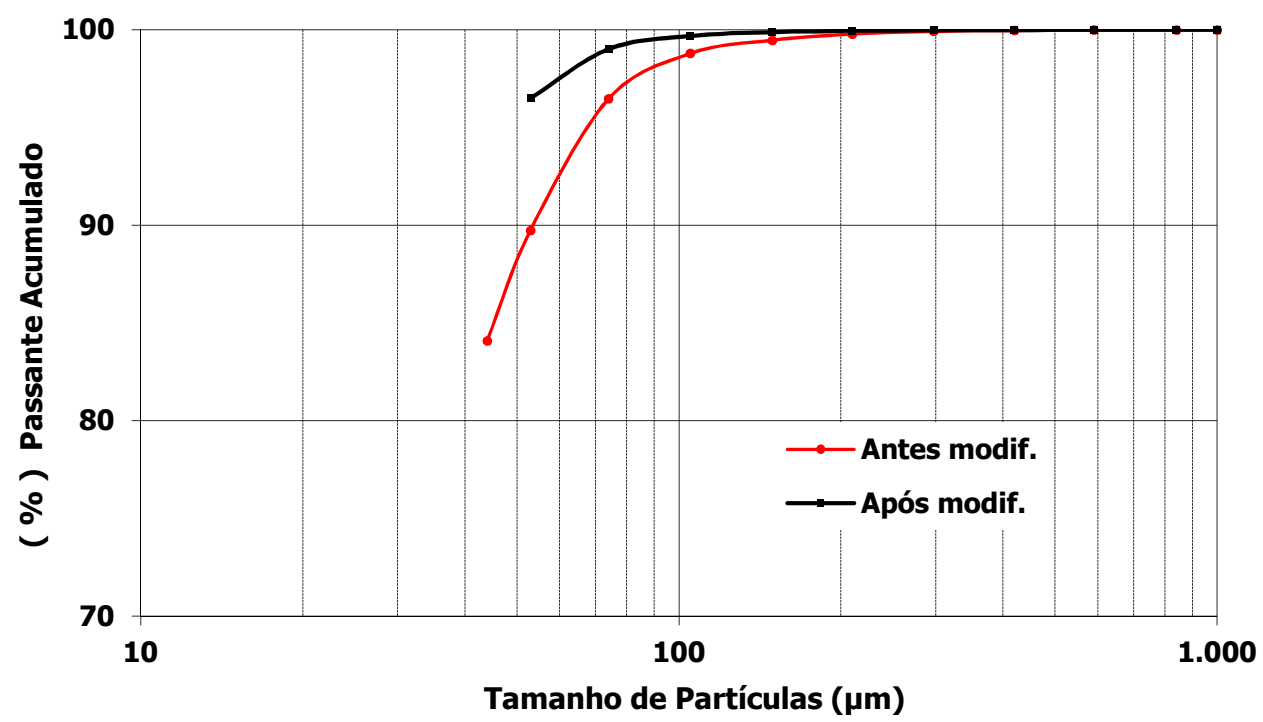

Figura 6. Distribuição granulométrica no overflow dos ciclones secundários antes e após a modificação do circuito.

O impacto na flotação pode ser observado tanto nos testes de flotação em bancada quanto nos dados coletados na alimentação da etapa de flotação subsequente a etapa de remoagem. A figura 07 apresenta os resultados dos testes de flotação em bancada realizados com amostras da alimentação o dos moinhos secundários. Os testes foram realizados variando a granulometria da amostra (expresso em termos do percentual maior que 200\#). Como pode ser observado na figura, quanto maior a granulometria da amostra (maior o percentual retido em 200\#, maior é o teor de $\mathrm{SiO}_{2}$ no concentrador da flotação. 


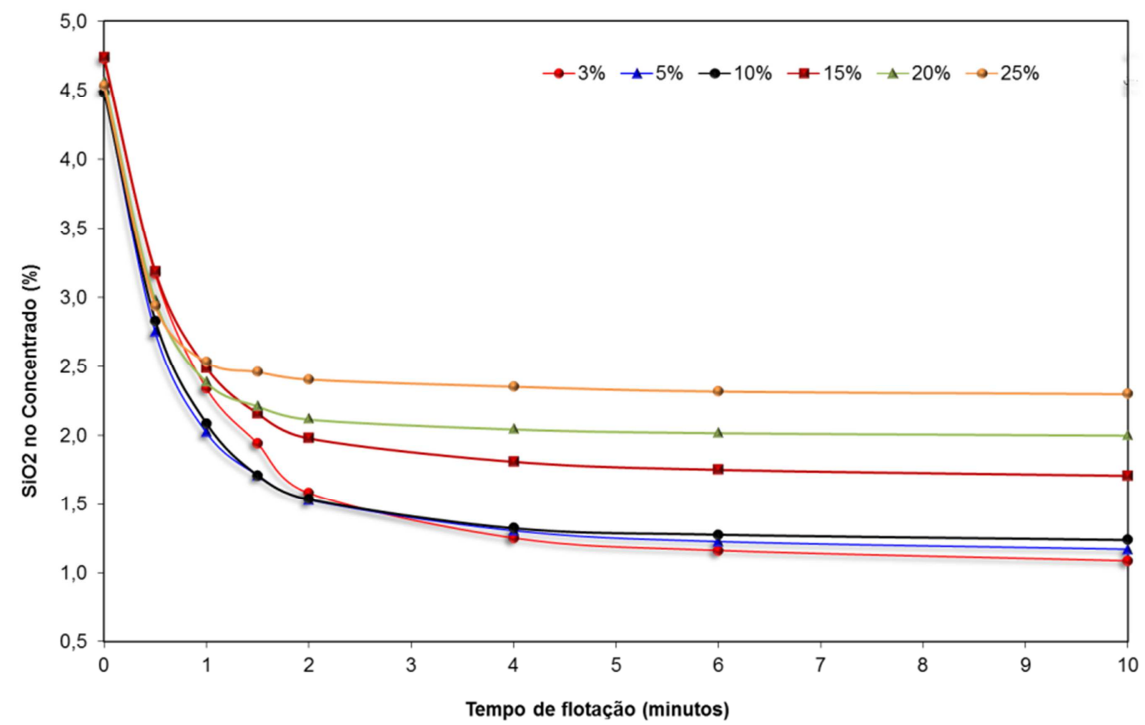

Figura 7. resultados dos testes de flotação em bancada realizados com amostras da alimentação dos moinhos secundários. Os testes foram realizados variando a granulometria da amostra (expresso em termos do percentual maior que 200\#).

As figuras 08 e 09 apresentam os valores do grau de liberação e do percentual passante em $+200 \#$ medidos na alimentação da etapa de flotação antes e depois da modificação do circuito de remoagem. Como pode ser observado, o grau de liberação é maior e o percentual maior que 200\# é menor após a modificação do circuito.

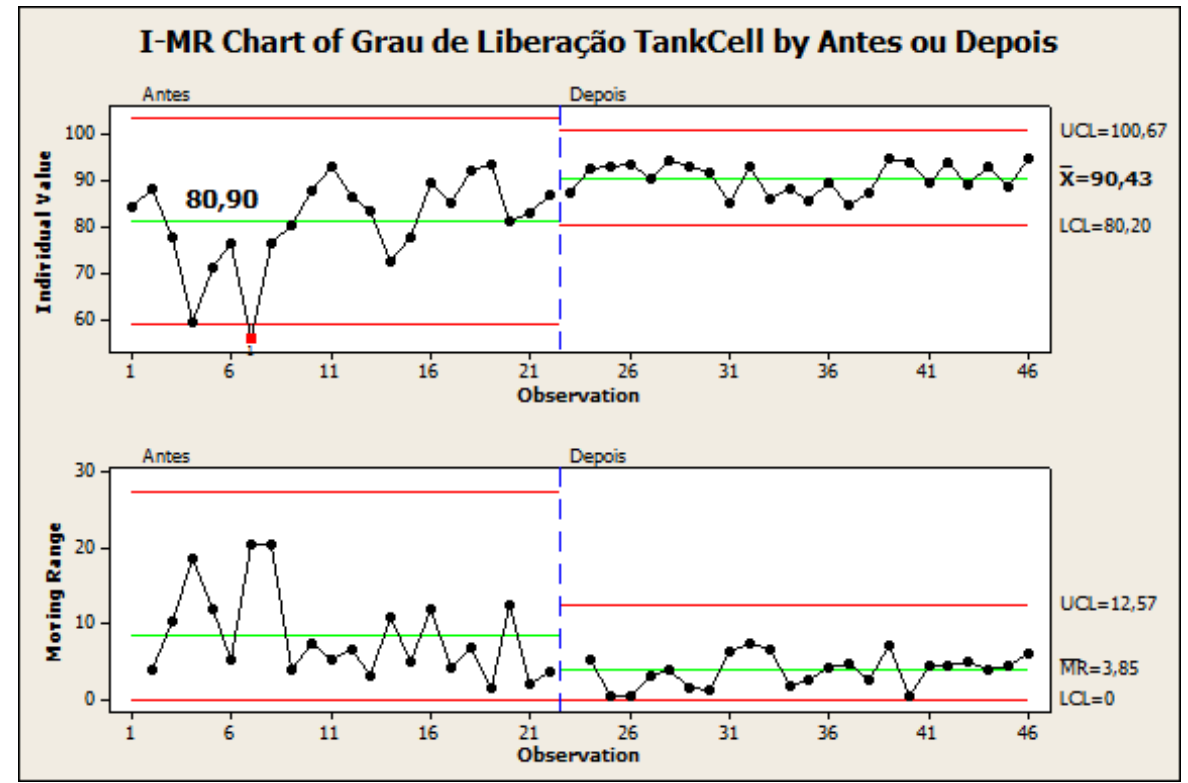

Figura 8. Grau de liberação medido na alimentação da etapa de flotação antes e depois da modificação do circuito de remoagem. 


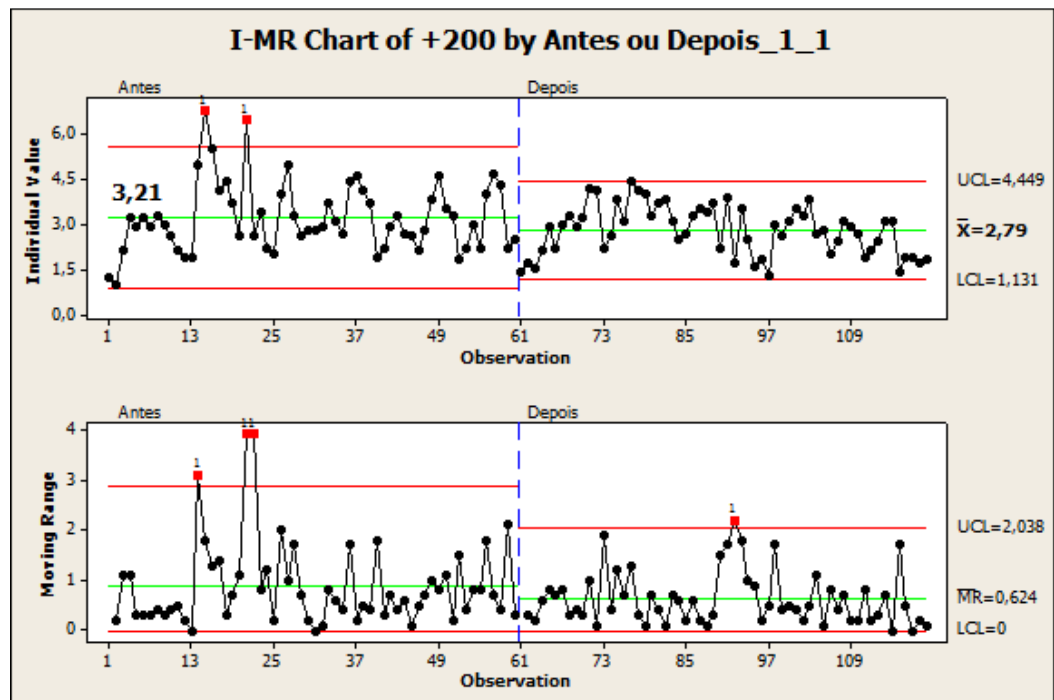

Figura 9. Percentual passante em +200\# medido na alimentação da etapa de flotação antes e depois da modificação do circuito de remoagem.

\section{CONCLUSÃO}

As alterações realizadas que consistiram em moer todo o pré-concentrado em circuito fechado reverso e não mais em dois estágios, sendo um em circuito aberto e outro em circuito fechado permitiu aumentar a eficiência energética do circuito de remoagem com redução de $9,3 \%$ no consumo de energia, redução do percentual de material fino no interior do moinho e adequação da granulometria e grau de liberação para a etapa subsequente de flotação. Além disso, também foi possível reduzir o número de intervenções para manutenção nos ciclones secundários.

\section{REFERÊNCIAS}

1 Donda, J.D. Um Método para Prever o Consumo de Energia na (Re)Moagem de Concentrados de Minérios de Ferro em Moinhos de Bola. [Tese, Doutorado em Engenharia Metalúrgica e de Minas]. Escola de Engenharia da UFMG, Belo Horizonte; 2003.

2 Ballantune, G R, Powell M S. Benchmarkin Comminution Energy Consumption for the Processing of Copper and Gold Ores. Minerals Engineering 2014; 65;109-114.

3 Bond, F. C. Crushing and Grinding Calculations. Additions and Revisions. AllisChalmers Manufacturing Co. Milwaukee, Wiscosin. April, 1962.

4 Chen X, Peng Y, Bradshaw D. The Effect of Particle Breakage Mechanisms During Regrinding on the Subsequent Cleaner Flotation. Minerals Engineering 2014; 6668;157-164.

5 Jankovic A, Valery W. Closed Circuit Ball Mill - Basic Revisited. Minerals Engineering 2013; 43-44;148-153.

6 Nadolski S, Klein B, Kumar A, Davaanyam Z. An Energy Benchmarking Model for Mineral Comminuition. Minerals Engineering 2014; 65;178-186.

7 Pourghahramani P, Dehgahani H, Sam A. A new Approach for Evaluating the Performance of Industrial Regrinding Tumbling Ball Mills Based on Grindability an Flotability. Minerals Engineering 2013; 49;116-120.

8 Sayadi A R, Khalesi M R, Borji M K. A Parametric Cost Model for Mineral Grinding Mills. Minerals Engineering 2014; 55;96-102. 